ARTICLE

Received 12 Dec 2013 | Accepted 27 Jan 2014 | Published 18 Feb $2014 \quad$ DOl: 10.1038/ncomms4328

\title{
Optical signatures of silicon-vacancy spins in diamond
}

Tina Müller ${ }^{1, \star}$, Christian Hepp ${ }^{2, \star}$, Benjamin Pingault ${ }^{1}$, Elke Neu ${ }^{2,3}$, Stefan Gsell ${ }^{4}$, Matthias Schreck ${ }^{4}$, Hadwig Sternschulte ${ }^{5,6}$, Doris Steinmüller-Nethl ${ }^{5}$, Christoph Becher ${ }^{2}$ \& Mete Atatüre ${ }^{1}$

Colour centres in diamond have emerged as versatile tools for solid-state quantum technologies ranging from quantum information to metrology, where the nitrogen-vacancy centre is the most studied to date. Recently, this toolbox has expanded to include novel colour centres to realize more efficient spin-photon quantum interfaces. Of these, the silicon-vacancy centre stands out with highly desirable photonic properties. The challenge for utilizing this centre is to realize the hitherto elusive optical access to its electronic spin. Here we report spin-tagged resonance fluorescence from the negatively charged silicon-vacancy centre. Our measurements reveal a spin-state purity approaching unity in the excited state, highlighting the potential of the centre as an efficient spin-photon quantum interface.

\footnotetext{
${ }^{1}$ Cavendish Laboratory, University of Cambridge, JJ Thomson Avenue, Cambridge CB3 OHE, UK. ${ }^{2}$ Fachrichtung 7.2 (Experimentalphysik), Universität des Saarlandes, Campus E2.6, 66123 Saarbrücken, Germany. ${ }^{3}$ Departement Physik, Universität Basel, Klingelbergstrasse 82,4056 Basel, Switzerland.

${ }^{4}$ Experimentalphysik IV, Institut für Physik, Universität Augsburg, Universitätsstrasse 1 Nord, 86159 Augsburg, Germany. ${ }^{5}$ DiaCoating GmbH, Mitterweg 24 , 6020 Innsbruck, Austria. ${ }^{6}$ Fakultät für Physik, Technische Universität München, James-Franck-Strasse 1, 85748 Garching, Germany. ${ }^{\star}$ These authors contributed equally to this work. Correspondence and requests for materials should be addressed to M.A. (email: ma424@cam.ac.uk).
} 
$\mathrm{D}$ iamond is a promising spin-free material for a range of novel technologies that make use of its optically accessible lattice impurities (colour centres). The most eminent colour centre in diamond to date, the negatively charged nitrogen-vacancy (NV) centre, has attracted great interest in recent years for quantum information processing ${ }^{1-3}$, electromagnetic field sensing ${ }^{4-7}$ and biomarking ${ }^{8,9}$. The NV centre owes its success to the optically accessible spin triplet in the ground state with room temperature electron-spin coherence times of up to $2 \mathrm{~ms}$ in ultrapure bulk diamond ${ }^{10}$. This allows for initialization ${ }^{11}$, coherent control ${ }^{12-15}$ and projective read-out ${ }^{11}$ to be performed with high fidelity. However, for photon-based quantum communication architectures, the challenge for the NV centre and most other diamond colour centres is to overcome the fundamental limitation of only weak emission into the zero-phonon line (ZPL), for example, by incorporating them in nanophotonic cavities ${ }^{16}$.

An alternative approach is to identify other centres with superior photonic (but comparable spin) properties to NV. Recent studies of such centres include chromium- ${ }^{17,18}$, xenon- ${ }^{19}$ nickel- ${ }^{20}$ and possibly oxygen-related centres ${ }^{21}$, as well as the negatively charged silicon-vacancy $(\mathrm{SiV})$ centre $^{22-24}$. The latter has the advantage of being the brightest reported colour centre in diamond $^{24}$. Its emission is concentrated to about $80 \%$ into its ZPL even at room temperature ${ }^{23}$, thus making it a promising single-photon source. One major challenge is to achieve direct (optical) access to the electronic spin degree of freedom similar to that of NVs. Previous electron-spin resonance measurements on ensembles of neutrally charged $\mathrm{SiV}$ centres have identified an $S=1$ ground state $e^{25-27}$, suggesting strongly that the negatively charged centres should be associated with an $S=1 / 2$ ground state. However, optical access to this electronic spin has remained elusive for both charge states.

Here, we report the direct accessing and spin-selective population of the negatively charged $\mathrm{SiV}$ centre excited states, achieved via resonant excitation and fluorescence under a magnetic field. In low-strain bulk diamond spin-selective excitation under finite magnetic field reveals a spin purity approaching unity in the excited state. We also investigate the effect of strain on the centres in nanodiamonds and discuss how spin selectivity in the excited state remains accessible in this regime.

\section{Results}

Characterization of the $\mathrm{SiV}^{-}$centre in a magnetic field. The $\mathrm{SiV}$ centre in diamond consists of a silicon atom and a vacancy in a split-vacancy configuration ${ }^{28-30}$, replacing two neighbouring carbon atoms in a diamond matrix along the $\langle 111\rangle$ axes (see Fig. 1a, inset). The substitutional silicon atom relaxes to the a

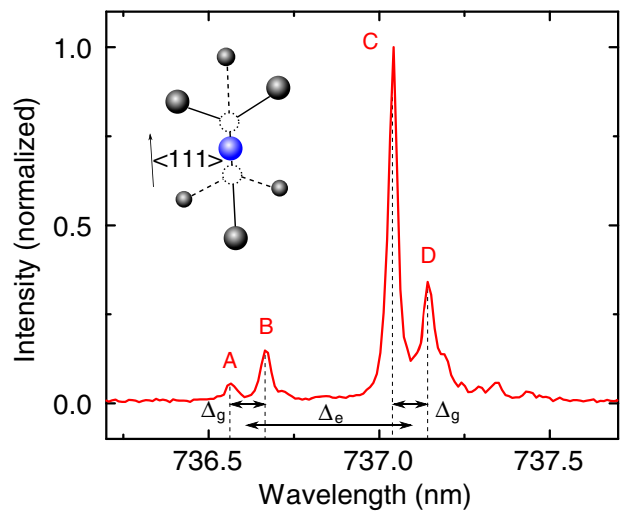

b

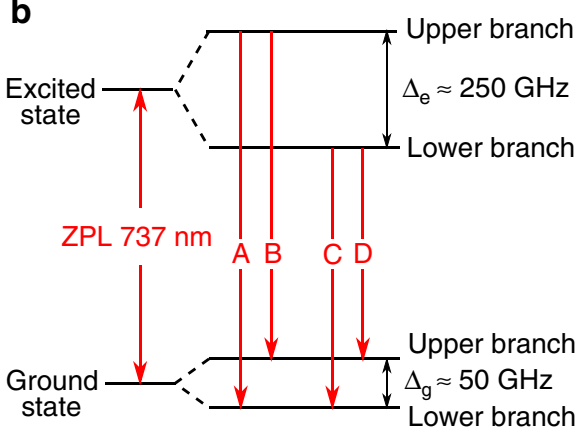

c

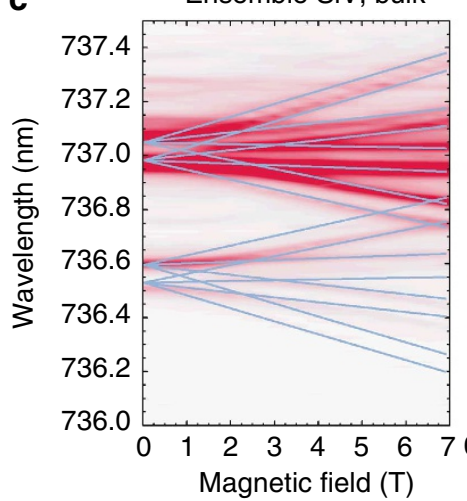

Single SiV, NC



d

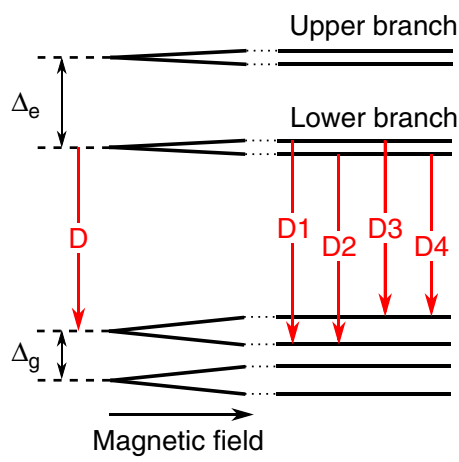

Figure 1 | Introduction to the $\mathbf{S i V}^{-}$centre. (a) Fluorescence spectrum at $4 \mathrm{~K}$ for an $\mathrm{SiV}^{-}$ensemble in [001]-oriented bulk diamond obtained by non-resonant excitation at $700 \mathrm{~nm}$. The dominant transitions due to the ${ }^{28} \mathrm{Si}$ isotope, forming two doublets, are labelled from $A$ to $D$, and the weaker, red-shifted transitions originate from ${ }^{29} \mathrm{Si}$ and ${ }^{30} \mathrm{Si}$. The atomic structure of the $\mathrm{SiV}^{-}$centre is shown in the inset, with the silicon atom (blue) in split-vacancy configuration between the unoccupied lattice sites (dashed circles) and the nearest neighbour carbon atoms (grey). (b) Energy level scheme of the $\mathrm{SiV}^{-}$centre. The split ground and excited states give rise to four optical transitions, labelled from $\mathrm{A}$ to $\mathrm{D}$ according to the transitions in $\mathbf{a}$. (c) Fluorescence spectra at $4 \mathrm{~K}$ as a function of magnetic field along [001] for an ensemble of SiV ${ }^{-}$centres in bulk diamond (red colour plot, light blue lines are a guide to the eye) and for a single $\mathrm{SiV}^{-}$in a diamond nanocrystal (blue colour plot), where the strain induces a larger splitting of the doublets at zero field, resulting in a clearer splitting into a quadruplet for each transition. Avoided crossings in the spectrum, marked by black dashed circles, originate from spin-orbit coupling ${ }^{31}$. (d) Magnetic field splitting of each spin-1/2 energy level. The optical transitions split into four as shown for transition $D$ due to the different g-factors in the ground and excited states. 
middle of the two lattice sites creating an inversion symmetric complex ${ }^{29,31}$. A total of 10 electrons are associated with this centre, as can be counted from the $\mathrm{SiV}$ structure in Fig. 1a, inset. One extra electron is captured to form the negatively charged complex $\left(\mathrm{SiV}^{-}\right)$. With 11 electrons in total, a single electron remains unpaired resulting in an effective spin-1/2 for the negatively charged state of the centre.

We study two types of samples containing $\mathrm{SiV}^{-}$centres: a diamond film grown homoepitaxially via chemical vapour deposition (CVD) on a high pressure high temperature bulk diamond $^{32}$ and CVD-grown diamond nanocrystals on an Ir substrat $^{23}$. The former offers a relatively homogenous low-strain environment preserving the intrinsic symmetry of the centre. On the other hand, the latter, from which the $\mathrm{SiV}^{-}$fluorescence can be extracted with high efficiency ${ }^{24}$, provides a strained environment modifying the emission properties from centre to centre $^{24,32}$.

At cryogenic temperatures, the photoluminescence spectrum of the ZPL reveals a characteristic fine structure composed of four transitions around $737 \mathrm{~nm}$, as displayed in Fig. 1a (transitions labelled from $A$ to $D$ ). This spectral signature originates from the doubly split ground and excited states shown in Fig. $1 b^{33,34}$. An applied magnetic field further lifts any degeneracies of these four transitions, as can be seen in Fig. 1c for a single $\mathrm{SiV}^{-}$centre in a nanocrystal (blue spectrum), and for an ensemble of $\mathrm{SiV}^{-}$ centres in bulk diamond (red spectrum). Common to both spectra is the measured quadruplet splitting in the optical transitions, which is consistent with an energy level scheme based on spin-1/2 ground and excited states (Fig. 1d) ${ }^{35}$. Theoretical analysis based on density functional theory ${ }^{28}$, as confirmed by a recent $a b$ initio study ${ }^{36}$, has determined a $D_{3 \mathrm{~d}}$ symmetry for the $\mathrm{SiV}^{-}$centre and assigned the optical ground and excited states to $E_{\mathrm{g}}$ and $E_{\mathrm{u}}$ states, respectively. The system can then be described by a Hamiltonian comprising orbital and spin Zeeman terms, the Jahn-Teller effect, which partially lifts the orbital degeneracies and a spin-orbit coupling term $(\boldsymbol{L} \cdot \boldsymbol{S})$. Group theoretic analysis predicts that, in the case of the $\mathrm{SiV}^{-}$centre, only the $L_{z} S_{z}$ component ( $\mathrm{z}$ along the $\mathrm{C}_{3}$ axis of the centre, that is, the [111] axis of the lattice) of the spin-orbit operator acts on the $E_{\mathrm{g}}$ and $E_{\mathrm{u}}$ states $^{31}$, providing an inherent quantization axis along the $\langle 111\rangle$ directions but leaving the spin as a good quantum number analogous to the $\mathrm{NV}^{-}$centres $^{37}$. The degree of spin mixing introduced by the Jahn-Teller and Zeeman parts of the Hamiltonian can be quantified by defining spin purity as the probability of finding a state in one given spin projection $m_{\mathrm{s}}= \pm 1 / 2$ only.

Resonance fluorescence at $0 \mathrm{~T}$. Resonance fluorescence through state-selective excitation is a powerful tool to reveal the internal structure of quantum emitters. We first study an ensemble of $\mathrm{SiV}^{-}$centres in a low-strain bulk diamond at $0 \mathrm{~T}$. Figure $2 \mathrm{a}$ displays the $\mathrm{SiV}^{-}$spectrum (red curve) observed when driving transition B resonantly (as labelled in Fig. 1a). Here the laser is suppressed by polarization rejection (see Methods) and contributes only a small fraction to the full spectrum (see Methods and Supplementary Fig. 1). Although resonant excitation selectively populates one excited state branch, all four transitions are visible indicating a relaxation process between the excited state branches before photon emission. Tuning the laser frequency across transition A, while monitoring the fluorescence of transition $\mathrm{C}$ in the spirit of photoluminescence excitation, reveals the absorption profile of transition A (solid red circles in Fig. 2b). The extracted full width at half maximum of $\sim 10 \mathrm{GHz}$ is consistent with the inhomogenous broadening of the ensemble under non-resonant excitation ${ }^{32}$ due to residual strain in the sample (as evidenced by the shift of transitions along with laser frequency in Fig. 2c). For a single centre in a nanodiamond, the fluorescence spectrum, obtained by driving transition A resonantly, is shown in Fig. $2 \mathrm{~d}$ (blue curve). Owing to the strain in the crystal, the transitions of this centre are shifted beyond the inhomogenous broadening of the ensemble in bulk diamond, such that the exact spectrum varies from centre to centre. The absorption linewidth of $1.4 \mathrm{GHz}$ for this transition (Fig. 2e) is only an order of magnitude above the radiatively broadened limit $(\sim 100 \mathrm{MHz})^{22,33}$, which should be reachable straightforwardly using impurity-free diamond substrates, as was shown for NV centres ${ }^{38}$. This linewidth also marks the minimum Zeeman splitting needed to resolve spin sublevels spectrally under resonant excitation.

Spin-tagged fluorescence of unstrained $\mathrm{SiV}^{-}$centres. Applying a magnetic field of $4 \mathrm{~T}$ to the ensemble of $\mathrm{SiV}^{-}$centres in bulk diamond allows us to address excited states with a given spin orientation selectively. First, we drive the transition labelled A2 (see Supplementary Fig. 2) to populate a Zeeman sublevel of the upper branch of the excited state, expected theoretically to be a spin-up projection, as shown in Fig. 3a (ref. 31). The resulting spectrum, shown in Fig. 3b (red shaded curve), is strikingly different from the spectrum obtained under non-resonant excitation (Fig. 3c); only half of the available optical transitions are visible and they originate from two excited states only. This is in stark contrast to thermal distribution at $4 \mathrm{~K}$, which would lead to a finite population probability for all excited states. Here the relaxation process in the excited state takes place only between the two sublevels with the same Zeeman response, that is, same spin projection. To populate an excited state sublevel with the opposite Zeeman response, expected theoretically to be a spindown projection, we resonantly drive transition B3 (blue double arrow in Fig. 3d). The resulting spectrum, shown in Fig. 3e (blue shaded curve), is strongly anti-correlated with that in Fig. 3b, and the sum of the two spectra produces the full spectrum observed under non-resonant excitation (Fig. 3c). The spectra in Fig. 3b,e further reveal that from the populated excited states, optical transitions to all ground states occur, irrespective of their spin projection. The anti-correlation in the spectra arises from the high degree of spin purity in the excited state, that is, a high probability of finding each excited state in one of the two spin states only. In contrast, what appears as a violation of spin preservation in the optical transitions is a consequence of the orientation of the applied magnetic field affecting mostly the electronic ground state. Indeed, when the magnetic field is not parallel to the $\mathrm{SiV}^{-}$axis (as is the case in our experiment, see Methods) the original quantization axis of the centre is tilted because of the magnetic field-induced non-diagonal terms in the spin-orbit basis, which in turn induces an apparent spin mixing. With an angle of $54.7^{\circ}$ between the $\langle 111\rangle \mathrm{SiV}^{-}$axes and the 4$\mathrm{T}$ magnetic field along [001] (see Supplementary Fig. 3), the spinorbit coupling strength in the excited state is still dominant and the spin-projection sublevel in the higher-lying excited state sustains a spin purity of $97 \%$ (Hepp et al. ${ }^{31}$ ), which agrees well with the $4.1 \pm 1.2 \%$ overlap between the two anti-correlated spectra of Fig. 3 (see Methods and Supplementary Fig. 4). On the other hand, the spin-orbit coupling strength in the ground state is comparable to the off-axis contributions of the Zeeman Hamiltonian (at $4 \mathrm{~T}$ ) resulting in a degree of spin purity ranging from 50 to $80 \%$ for the ground-state branches ${ }^{31}$. This effective spin mixing gives rise to finite intensity in all optical transitions starting from a given excited state.

Spin-tagged fluorescence of strained single $\mathrm{SiV}^{-}$centres. We now investigate the influence of strain through resonance 

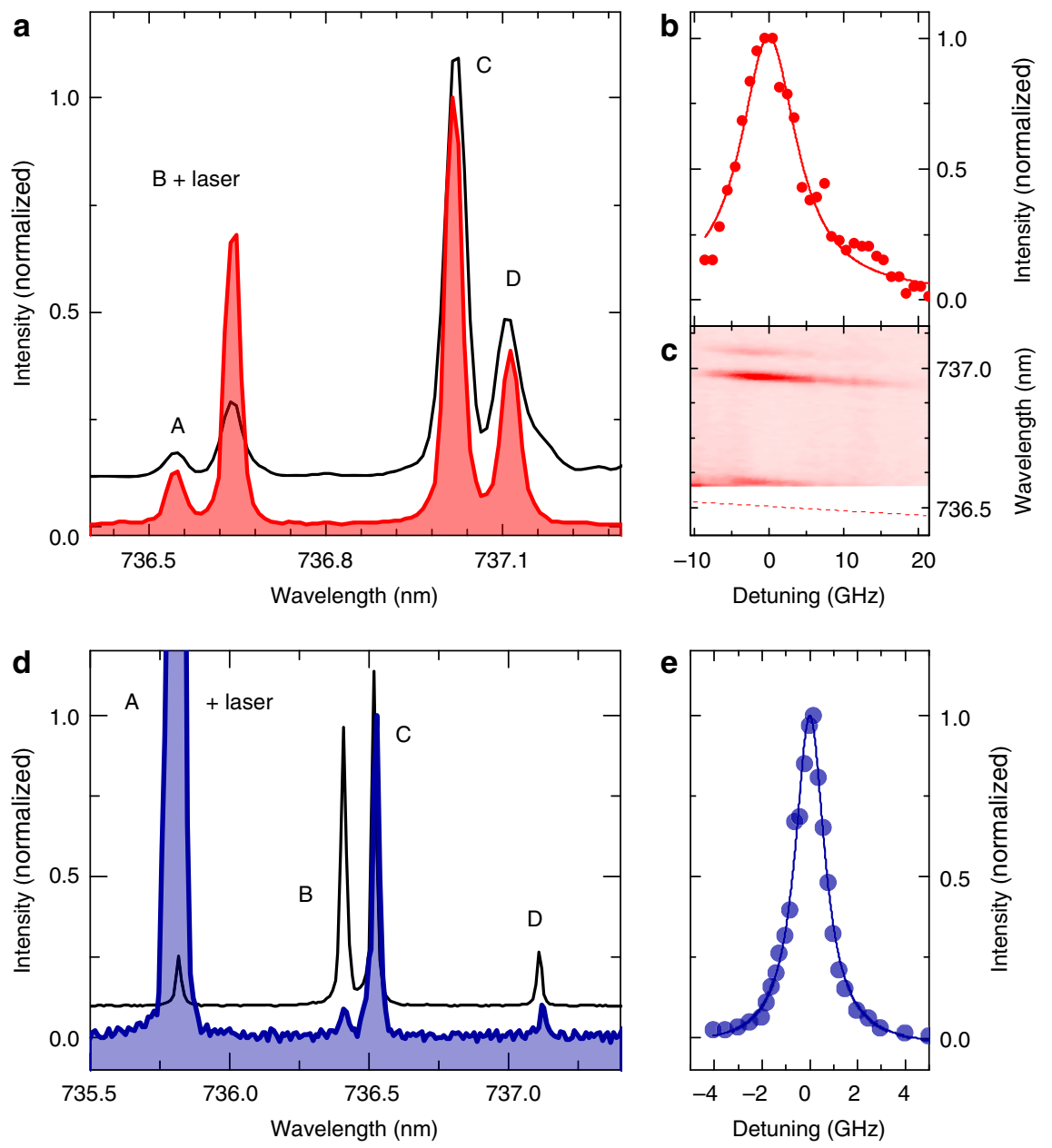

Figure 2 | Resonance fluorescence at $\mathbf{0 T}$. (a) Resonance fluorescence spectrum at $4 \mathrm{~K}$ for a SiV ${ }^{-}$ensemble (red shaded curve) with laser resonantly driving transition B, and non-resonant spectrum (black solid curve). (b) Photoluminescence excitation of transition $\mathrm{A}^{\text {for a SiV }}{ }^{-}$ensemble. The maximum intensity of transition $\mathrm{C}$ is plotted as a function of the resonant laser detuning (red dots) and can be fitted by a single Lorentzian (red solid curve) with full width at half maximum (FWHM) $9.1 \pm 0.7 \mathrm{GHz}$. (c) As the laser is tuned towards lower wavelengths across transition $\mathrm{A}$ (red dashed line), the observed fluorescing transition wavelengths B-C exhibit the same blue shift in the spectrum. Consequently, for a given laser frequency only a subensemble of the centres with identical strain conditions is excited. (d) Resonance fluorescence spectrum at $4 \mathrm{~K}^{\text {for a single SiV }}{ }^{-}$in nanodiamond (confirmed via photon correlation measurements), with laser resonantly exciting transition A (blue shaded curve) and non-resonant spectrum (black solid curve). The relative intensities of transitions under resonant excitation differ from the ones obtained in the non-resonant spectrum owing to the polarization-based laser suppression (see Supplementary Fig. 5). (e) Photoluminescence excitation of transition A for the same single centre in nanodiamond. The maximum intensity of peak $\mathrm{C}$ is plotted as a function of laser detuning (blue dots) and can be fitted by a single Lorentzian (blue solid curve) with FWHM of $1.4 \pm 0.1 \mathrm{GHz}$.

fluorescence from a single centre located in a nanodiamond. A magnetic field of $2 \mathrm{~T}$ allows us to optically resolve the individual transitions of the centre shown in Fig. 1c. The excitation laser is brought into resonance with transition A1 (as shown in Fig. 4a) and transition A2. This leads to fluorescence spectra with selective population of the spin-up (blue curve) and spin-down (red curve) sublevels in the higher branch of the excited state, respectively, as seen in Fig. 4b. The signature of spin selectivity demonstrated in bulk is only partially observed in the resulting fluorescence spectra shown in Fig. 4b. While the transitions B1$\mathrm{B} 4$, originating directly from the higher-lying branch of the excited state, still exhibit a high degree of spin selectivity (hence spin purity), the transitions $\mathrm{C} 1-\mathrm{D} 4$, which originate from the lower branch after a relaxation step, do not display such spin selectivity. This breakdown is induced by the strong strain field not oriented with the symmetry axis of the centre. This can be understood based on the model presented in (Hepp et al. ${ }^{31}$ ), where an additional term for the strain perturbation is added to the total Hamiltonian to account for the strain field in the nanodiamonds (not discussed here).

Bypassing the interbranch relaxation mechanism by directly populating a spin sublevel of the lower branch allows us to access the degree of spin purity in this branch. This is illustrated in Fig. $4 \mathrm{c}$ for the case of populating the spin-up sublevel. Phononassisted excitation to the upper branch is strongly suppressed at $4 \mathrm{~K}$ owing to the large energy difference between the two branches for this centre. In the resulting spectra, shown in Fig. $4 \mathrm{~d}$, the transitions resonantly populating spin-up and spin-down sublevels are indicated by blue and red arrows, respectively. Under these conditions, the contrast between fluorescence intensity originating from the two spin orientations (red and blue filled curves) is recovered to above $90 \%$. This evidences highspin purity within both excited state branches. Therefore, by selecting the driven transitions, spin-selective optical access to $\mathrm{SiV}^{-}$centres can be achieved in strained nanodiamonds as well as low-strain bulk diamond. 



Figure 3 | Resonance fluorescence of ensemble SiV ${ }^{-}$at $4 \mathbf{T}$. (a) Energy level scheme for a SiV ${ }^{-}$in bulk diamond at 0 and $4 \mathrm{~T}$. The driven transition populates the spin-down projection in the upper branch of the excited states and is indicated by a red double arrow. Relaxation takes place to the spin-down projection in the lower branch of the excited states as indicated by the green arrow. The thin red arrows indicate the transitions observed in the spectrum in panel b. (b) Resonance fluorescence spectrum measured driving transition A2 at 4 T for an SiV ${ }^{-}$ensemble in bulk diamond (red shaded curve). (c) Non-resonant fluorescence spectrum at $4 \mathrm{~T}_{\text {for }}$ an $\mathrm{SiV}^{-}$ensemble (black solid curve). (d) Energy level scheme for a SiV ${ }^{-}$in bulk diamond at 0 and $4 \mathrm{~T}$. The driven transition populates the spin-up projection in the upper branch of the excited state and is indicated by a blue double arrow. Relaxation takes place to the spin-up projection in the lower branch of the excited state as indicated by the green arrow. The thin blue arrows indicate the transitions observed in the resonant spectrum (e). (e) Resonance fluorescence spectrum measured when driving transition B3 at $4 \mathrm{~T}$ for an SiV ${ }^{-}$ensemble (blue shaded curve). The fluorescing transitions in $\mathbf{b}$ and $\mathbf{e}$ are highlighted with red and blue panels, respectively, on the non-resonant spectrum in $\mathbf{c}$. The laser wavelengths used for resonant excitation in $\mathbf{b}$ and $\mathbf{e}$ are indicated with red and blue dashed lines, respectively.

\section{Discussion}

A natural extension of this work is to align the $C_{3}$ symmetry axis of a $\mathrm{SiV}^{-}$centre to the magnetic field either by rotating the samples (which was technically not possible in our set-up) or by implanting $\mathrm{SiV}^{-}$centres in a [111]-oriented diamond crystal. Both approaches are expected to restore more than $90 \%$ spin purity in the ground state and near-unity spin purity in the excited state eliciting the inherent optical selection rules linked to the spin orientation. These properties mark the $\mathrm{SiV}^{-}$centre desirable for all applications that require optical access to welldefined spin states. All-optical ultrafast spin manipulation techniques or optically detected magnetic resonance can then give access to the electronic spin coherence of the pure ground state. Full coherent control of the $\mathrm{SiV}^{-}$spin state along with fluorescence-detection-based spin initialization and read-out will be within reach for the realization of a highly efficient spin-photon quantum interface.

\section{Methods}

Sample fabrication. The ensemble of $\mathrm{SiV}^{-}$centres in bulk was grown using a hot filament chemical vapour deposition technique ${ }^{33}$, where $\mathrm{SiV}^{-}$centres are created in situ owing to residual silicon contamination of the CVD reactor. A Ib high pressure high temperature diamond (Sumitomo) with [001] orientation was used as a substrate, and was overgrown with a high-quality diamond film. The homoepitaxial growth minimizes stress arising from thermal expansion mismatch of substrate and diamond, and helps reduce dislocations in the grown diamond lattice. High crystalline quality is achieved by applying optimized growth conditions involving a low methane fraction $\left(0.26 \% \mathrm{CH}_{4}\right.$ in $\left.\mathrm{H}_{2}\right)$ and slow growth. The thickness of the diamond film of $80-100 \mathrm{~nm}$ was estimated by growing a diamond film on a non-diamond substrate with identical growth conditions where the thickness could easily be measured.
Single $\mathrm{SiV}^{-}$centres in nanodiamonds were CVD grown on a silicon substrate, which was covered by an intermediate yttria-stabilized-zirconia buffer layer and atop a $150-\mathrm{nm}$ iridium layer ${ }^{14}$. This iridium layer allows for optimized growth conditions, and reduces the amount of silicon incorporated into the nanodiamonds from the substrate during growth. Before growth, the substrate is seeded with deagglomerated synthetic nanodiamonds with sizes up to $30 \mathrm{~nm}$ (Microdiamant Liquid Diamond MSY). The aqueous solution of these diamonds is diluted appropriately to achieve a seed density of roughly 2.5 seeds per $\mu \mathrm{m}^{2}$. The density of crystals containing $\mathrm{SiV}^{-}$centres is estimated to be around one per $50 \times 50 \mu \mathrm{m}^{2}$. The seeded substrates were exposed to microwave plasma-assisted CVD process for $25 \mathrm{~min}$, using a hydrogen-plasma containing $1 \%$ methane. The gas pressure was $30 \mathrm{mbar}$ and the microwave power was $2,000 \mathrm{~W}$. These conditions resulted in a crystal size of about $130 \mathrm{~nm}$ with a s.d. of $30 \mathrm{~nm}$.

Confocal microscope set-up. The sample is mounted on a piezo-driven three-axis translation stage (attocube ANPz 101/LT and two ANPx 101/LT) in a helium bath cryostat $(T=4.2 \mathrm{~K})$ at the centre of a fixed orientation, tunable $7 \mathrm{~T}$ superconducting magnet (Cryogenic) in Faraday configuration. Excitation of the $\mathrm{SiV}^{-}$centres is performed non-resonantly at $700 \mathrm{~nm}$ (Coherent Mira 900-CW) and resonantly using a frequency-tunable external-cavity diode laser around $737 \mathrm{~nm}$ (Toptica DL 100 Pro Design). SiV ${ }^{-}$spectra were recorded using a spectrometer with $\sim 40 \mu \mathrm{eV}$ resolution (PI Acton). All measurements are performed using a fibre-based confocal microscope with polarization-controlled excitation and collection (polarizers: Thorlabs LPVIS050-MP, half-wave plates: Thorlabs AHWP05M-980). Light is focused onto the sample with a numerical aperture $=0.68$ aspheric lens (Thorlabs C330TME-B), and residual laser light in the detection arm is removed with either a 720-nm longpass filter (third millennium) or with a polarizer set perpendicularly to the incoming laser polarization. With the latter technique, laser suppression up to $5 \times 10^{-7}$ was reached.

Determination of the photon ratio. In the resonant spectrum of Fig. 2a, the integrated intensity of each transition is evaluated through Lorentzian fits (see Supplementary Fig. 1a with the following results: $I(\mathrm{~A})=0.14, I(\mathrm{~B})+I($ laser $)=0.66$, $I(\mathrm{C})=1.0, I(\mathrm{D})=0.46$ (the intensities are normalized to that of transition C. 
a

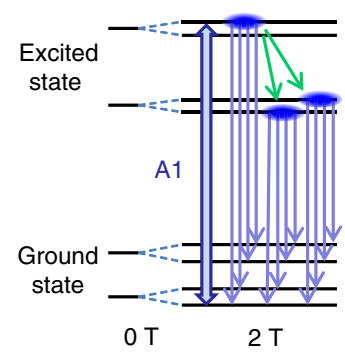

C

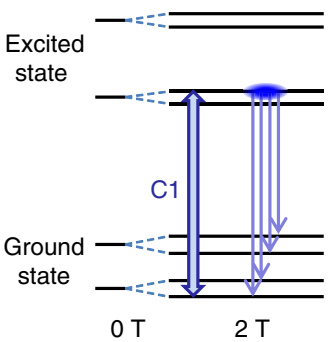

b
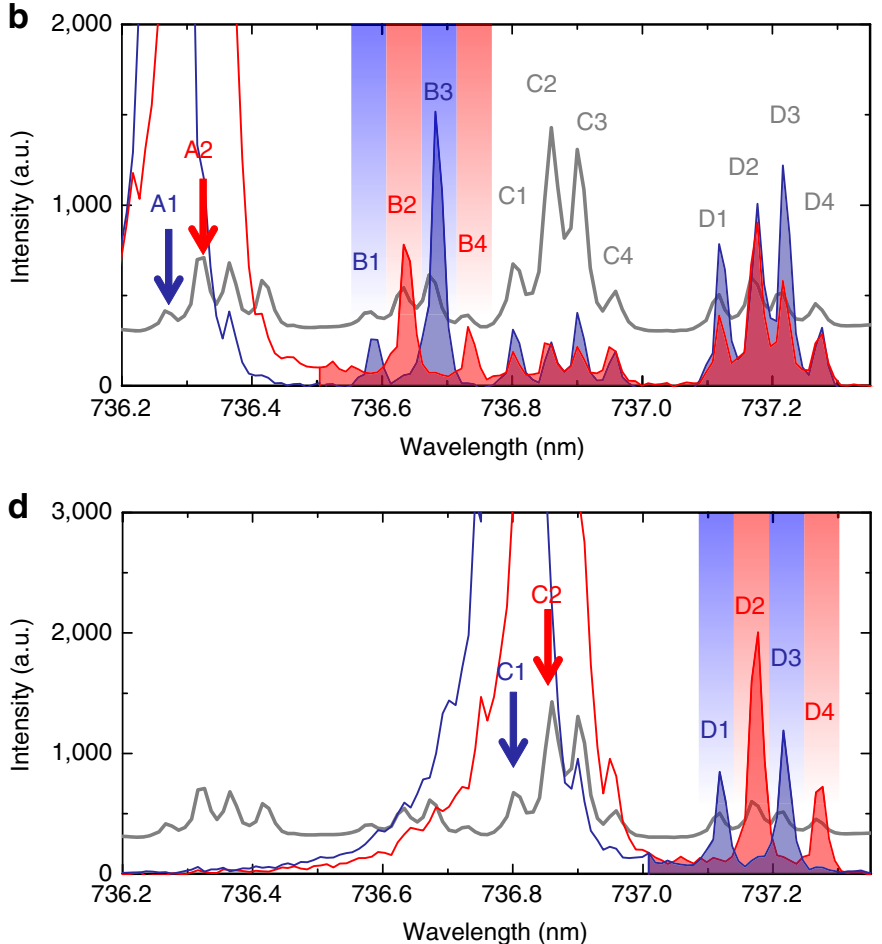

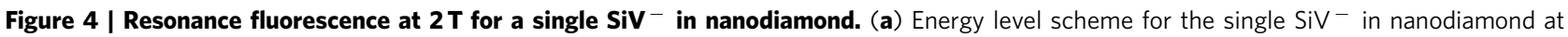
0 and $2 \mathrm{~T}$. Driving transition A1 (indicated by a blue double arrow) results in populating the spin-up sublevel in the upper branch of the excited state. From there, relaxation takes place to both lower branch sublevels (green arrows). The spectrum obtained when driving this transition is shown in panel b (blue shaded curve), along with a spectrum when resonantly driving transition A2, which populates the spin-down projection sublevel (red shaded curve). Driven transitions are indicated in the spectra by the red and blue arrows on the non-resonant spectrum (grey curve). (c) Same as panel a, but when driving transition C1 (blue double arrow), populating the spin-up sublevel in the lower branch. (d) The spectra obtained when driving $\mathrm{C} 1$ (blue shaded curve) and C2 (red shaded curve) along with the spectrum obtained under non-resonant excitation (grey curve).

Uncertainties on fits are below 0.01 for all transitions). First, a lower bound can be set by assuming that no emission from transition $B$ is collected $(I(B)=0)$. With this assumption, a ratio between laser and $\mathrm{SiV}^{-}$emission of 1:2.4 is obtained.

Next, we evaluate $I(B)$ when this transition is driven. The intensities in the resonant spectrum can be compared with the respective ones for non-resonant excitation (Fig. 1a), labelled as $I^{\prime}$. These are $I^{\prime}(\mathrm{A})=0.058, I^{\prime}(\mathrm{B})=0.20, I^{\prime}(\mathrm{C})=1.0$ and $I^{\prime}(\mathrm{D})=0.46$. The ratios $I / I^{\prime}$ differ for transitions $\mathrm{A}, \mathrm{C}$ and $\mathrm{D}$, with values ranging from 0.43 to 1.00 . Assuming that $I(\mathrm{~B}) / I^{\prime}(\mathrm{B})$ lies within that range, the intensity of transition $B$ in the resonant case can be expected between 0.20 and 0.47 . This leads to an intensity ratio of the laser to $\mathrm{SiV}^{-}$emission between the extrema of 1:3.9 and 1:11.

Another way of evaluating the intensity of transition B when resonantly driven is by applying the same method of intensity comparison with the spectrum where transition A is resonantly excited, knowing that transitions A and B share the same excited state. In the spectrum with A driven resonantly (see Supplementary Fig. $1 \mathrm{~b})$, the following intensities $I^{\prime \prime}$ are obtained: $I^{\prime \prime}(\mathrm{B})=0.33, I^{\prime \prime}(\mathrm{C})=1$ and $I^{\prime \prime}(\mathrm{D})=0.27$. The ratios $I / I^{\prime \prime}$ range from 0.59 to 1.00 , resulting in the intensity of transition $\mathrm{B}$ being between 0.33 and 0.57 . This finally leads to a ratio between laser and $\mathrm{SiV}^{-}$emission ranging from 1:5.9 to $1: 24$.

Contrast between transition subsets under excitation. Here we describe how we can extract information about the degree of spin conservation in the excited state by comparing transition intensities when populating spin-up and spin-down excited states by driving transitions A2 and B3, respectively (Fig. 3b,e). For this analysis, we restrict ourselves to intensities in transitions C1 to D4, originating from the lower-lying excited state. This is to exclude contributions from the excitation laser. Visual inspection of the spectra in Supplementary Fig. 4 (red curve for driving transition A2 and blue curve when driving transition B3) shows that when populating the spin-down excited states (red spectrum), contributions to transitions originating from spin-up excited states are within the noise of the spectrum. In contrast, when populating the spin-up excited states (blue spectrum), a finite contribution into the 'forbidden' transition $\mathrm{C} 2$ is visible. We use this intensity to estimate the branching ratio of the relaxation mechanism in the excited state.

We now consider the blue spectrum in Supplementary Fig. 4. According to Fermi's golden rule, the transition rate $\Gamma$ for a specific transition is given by $\Gamma=(2 \pi / \hbar) \rho_{i}\left|\left\langle\varphi_{i}|D| \varphi_{f}\right\rangle\right|^{2}$, where $\hbar$ is the reduced Planck's constant, $\rho_{\mathrm{i}}$ is the probability of finding the system in the excited state at the origin of the transition, $\phi_{\mathrm{i}}$ is the electronic wavefunction of the excited state, $D$ is the dipole operator and $\phi_{\mathrm{f}}$ is the electronic wavefunction in the ground state. If we assume that the dipole matrix element is constant for all transitions, then the transition rates become proportional to the populations $\rho_{\mathrm{i}}$ in the excited state and give access to the branching ratios between the two spin states. This assumption is not entirely correct, but since $\mathrm{C} 2$ is the brightest transition under non-resonant excitation, the transition matrix element will be larger. Consequently, the branching ratio will be overestimated when considering the blue spectrum yielding a lower limit for spin conservation

Focusing on the blue spectrum for the spin-down transitions, the transitions can be fitted with a series of five Lorentzians (blue shaded curve in Supplementary Fig. 4): four for the 'allowed' transitions C1, D1, C3 and D3, and one for the 'forbidden' transition C2. The other 'forbidden' transitions are too weak to be considered. The intensity ratio between transition $\mathrm{C} 2$ and the total emission into the five transitions is $3 \pm 1.2 \%$. The additional error made by neglecting the intensities into the forbidden transitions D2, C4 and D4 can be calculated by considering the respective intensities in the red spectrum. A Lorentzian fit of the four visible transitions reveals that transition $\mathrm{C} 2$ constitutes $73.2 \pm 0.9 \%$ of the total red spectrum. Therefore, the branching ratio between transitions C2, D2, C4 and D4 (the forbidden ones) and transitions C1, D1, C3 and D3 is $4.1 \pm 1.2 \%$, confirming the strong spin conservation of the relaxation mechanism in the excited state for centres in bulk diamond.

\section{References}

1. Wrachtrup, J. \& Jelezko, F. Processing quantum information in diamond. J. Phys. Condens. Matter 18, S807-S824 (2006).

2. Neumann, P. et al. Quantum register based on coupled electron spins in a room temperature solid. Nat. Phys. 6, 249-253 (2010).

3. Bernien, H. et al. Heralded entanglement between solid-state qubits separated by three metres. Nature 497, 86-90 (2013).

4. Degen, C. L. Scanning magnetic field microscope with a diamond single-spin sensor. Appl. Phys. Lett. 92, 243111 (2008).

5. Taylor, J. et al. High-sensitivity diamond magnetometer with nanoscale resolution. Nat. Phys. 4, 810-816 (2008).

6. Dolde, F. et al. Electric-field sensing using single diamond spins. Nat. Phys. 7, 459-463 (2011). 
7. Grinolds, M. S. et al. Nanoscale magnetic imaging of a single electron spin under ambient conditions. Nat. Phys. 9, 215-219 (2013).

8. Mohan, N. et al. In vivo imaging and toxicity assessments of fluorescent nanodiamonds in Caenorhabditis elegans. Nano Lett. 10, 3692-3699 (2010).

9. Hegyi, A. \& Yablonovitch, E. Molecular imaging by optically detected electron spin resonance of nitrogen-vacancies in nanodiamonds. Nano Lett. 13, 1173-1178 (2013)

10. Balasubramanian, G. et al. Ultralong spin coherence time in isotopically engineered diamond. Nat. Mater. 8, 383-387 (2009).

11. Robledo, L. et al. High-fidelity projective read-out of a solid-state spin quantum register. Nature 477, 574-578 (2011).

12. Jelezko, F., Gruber, T., Popa, I. \& Wrachtrup, J. Observation of coherent oscillations in a single electron spin. Phys. Rev. Lett. 92, 76401 (2004).

13. Childress, L. et al. Coherent dynamics of coupled electron and nuclear spin qubits in diamond. Science 314, 281-285 (2006).

14. Dutt, M. V. G. et al. Quantum register based on individual electronic and nuclear spin qubits in diamond. Science 316, 1312-1316 (2007).

15. Yale, C. G. et al. All-optical control of a solid-state spin using coherent dark states. Proc. Natl Acad. Sci. USA 110, 7595 (2013).

16. Faraon, A., Barclay, P. E., Santori, C., Fu, K.-M. \& Beausoleil, R. G. Resonant enhancement of the zero-phonon emission from a colour centre in a diamond cavity. Nat. Photon. 5, 301-305 (2011).

17. Siyushev, P. et al. Low-temperature optical characterization of a near-infrared single-photon emitter in nanodiamonds. New J. Phys. 11, 113029 (2009).

18. Aharonovich, I. et al. Two-level ultrabright single photon emission from diamond nanocrystals. Nano Lett. 9, 3191-3195 (2009).

19. Deshko, Y. \& Gorokhovsky, A. A. Spectroscopy and micro-luminescence mapping of Xe-implanted defects in diamond. Low Temp. Phys. 36, 465-471 (2010)

20. Lee, S.-Y. et al. Readout and control of a single nuclear spin with a meta-stable electron spin ancilla. Nat. Nanotechnol. 8, 487-492 (2013).

21. Nadolinny, V. A. et al. A study of ${ }^{13} \mathrm{C}$ hyperfine structure in the EPR of nickel-nitrogen-containing centres in diamond and correlation with their optical properties. J. Phys. Condens. Matter 11, 7357 (1999).

22. Wang, C., Kurtsiefer, C., Weinfurter, H. \& Burchard, B. Single photon emission from $\mathrm{SiV}$ centres in diamond produced by ion implantation. J. Phys. B 39, 37 (2006).

23. Neu, E. et al. Single photon emission from silicon-vacancy colour centres in chemical vapour deposition nano-diamonds on iridium. New J. Phys. 13, 025012 (2011)

24. Neu, E., Agio, M. \& Becher, C. Photophysics of single silicon vacancy centres in diamond: implications for single-photon emission. Opt. Express. 20, 19956-19971 (2012).

25. Iakoubovskii, K. \& Stesmans, A. Characterization of hydrogen and silicon-related defects in CVD diamond by electron spin resonance. Phys. Rev. B 66, 195207 (2002).

26. Edmonds, A. M., Newton, M. E., Martineau, P. M., Twitchen, D. J. \& Williams, S. D. Electron paramagnetic resonance studies of silicon-related defects in diamond. Phys. Rev. B 77, 245205 (2008).

27. D'Haenens-Johansson, U. F. S. et al. Optical properties of the neutral silicon split-vacancy centre in diamond. Phys. Rev. B 84, 245208 (2011)

28. Moliver, S. Electronic structure of neutral silicon-vacancy complex in diamond. Tech. Phys. 48, 1449-1453 (2003).
29. Goss, J. P., Briddon, P. R. \& Shaw, M. J. Density functional simulations of silicon-containing point defects in diamond. Phys. Rev. B 76, 075204 (2007).

30. Goss, J. P., Jones, R., Breuer, S. J., Briddon, P. R. \& Oberg, S. The twelve-line $1.682 \mathrm{eV}$ luminescence center in diamond and the vacancy-silicon complex. Phys. Rev. Lett. 77, 3041 (1996).

31. Hepp, C. et al. The electronic structure of the silicon-vacancy color center in diamond. Phys. Rev. Lett. 112, 036405 (2014).

32. Neu, E. et al. Low temperature investigations of single silicon vacancy colour centres in diamond. New J. Phys. 043005 15, (2013).

33. Sternschulte, H., Thonke, K., Sauer, T., Münzinger, P. C. \& Michler, P. 1.681-eV luminescence center in chemical-vapor-deposited homoepitaxial diamond films. Phys. Rev. B 50, 14554-14560 (1994).

34. Clark, C. D., Kanda, H., Kiflawi, I. \& Sittas, G. Silicon defects in diamond. Phys. Rev. B 51, 16681-16688 (1995).

35. Sternschulte, H. et al. Uniaxial stress and Zeeman splitting of the $1.681 \mathrm{eV}$ optical center in a homoepitaxial CVD diamond film. Diamond Rel. Mat. 4, 1189-1192 (1995).

36. Gali, A. \& Maze, J. R. An $a b$ initio study on split silicon-vacancy defect in diamond: electronic structure and related properties. Phys. Rev. B 88, 235205 (2013).

37. Maze, J. R. et al. Properties of nitrogen-vacancy centers in diamond: the group theoretic approach. New J. Phys. 13, 025025 (2011)

38. Tamarat, P. h. et al. Stark shift control of single optical centers in diamond. Phys. Rev. Lett. 97, 083002 (2006).

\section{Acknowledgements}

We gratefully acknowledge financial support by the University of Cambridge, the European Research Council (FP7/2008-2013)/ERC Grant agreement number 209636, FP7 Marie Curie Initial Training Network $S^{3} \mathrm{NANO}$ and funding from the Bundesministerium für Bildung und Forschung within the projects EPHQUAM (Contract number 01BL0903) and QuOReP (Contract number 01BQ1011). We thank J. Maze, V. Waselowski, A. Gali, J. Becker and C. Matthiesen for technical assistance and helpful discussions.

\section{Author contributions}

M.A. and C.B. conceived the concept behind the project. E.N., S.G., M.S., H.S. and D.S.-N. prepared the samples. T.M., C.H. and B.P. performed the experiments. All authors contributed to the technical discussions and the writing of the manuscript.

\section{Additional information}

Supplementary Information accompanies this paper at http://www.nature.com/ naturecommunications

Competing financial interests: The authors declare no competing financial interests

Reprints and permission information is available online at http://npg.nature.com/ reprintsandpermissions.

How to cite this article: Müller, T. et al. Optical signatures of silicon-vacancy spins in diamond. Nat. Commun. 5:3328 doi: 10.1038/ncomms4328 (2014). 